\title{
Analytical Modeling of a Ball Screw Feed Drive for Vibration Prediction of Feeding Carriage of a Spindle
}

\author{
Lei Zhang, ${ }^{1,2}$ Taiyong Wang, ${ }^{1,2}$ Songling Tian, ${ }^{1,2}$ and Yong Wang \\ ${ }^{1}$ School of Mechanical Engineering, Tianjin University, Tianjin 300072, China \\ ${ }^{2}$ Key Laboratory of Mechanism Theory and Equipment Design of Ministry of Education, Tianjin University, Tianjin 300072, China \\ ${ }^{3}$ School of Mechanical Engineering, Tianjin University of Commerce, Tianjin 300134, China \\ Correspondence should be addressed to Taiyong Wang; tywang@139.com
}

Received 30 July 2016; Revised 21 October 2016; Accepted 14 November 2016

Academic Editor: Jaromir Horacek

Copyright (C) 2016 Lei Zhang et al. This is an open access article distributed under the Creative Commons Attribution License, which permits unrestricted use, distribution, and reproduction in any medium, provided the original work is properly cited.

\begin{abstract}
An analytical modeling approach for ball screw feed drives is proposed to predict the dynamic behavior of the feeding carriage of a spindle. Mainly considering the rigidity of linear guide modules, a ball-screw-feeding spindle is modeled by a mass-spring system. The contact stiffness of rolling interfaces in linear guide modules is accurately calculated according to the Hertzian theory. Next, a mathematical model is derived using the Lagrange method. The presented model is verified by conducting modal experiments. It is found that the simulated results correspond closely with the experimental data. In order to show the applicability of the proposed mathematical model, parameter-dependent dynamics of the feeding carriage of the spindle is investigated. The work will contribute to the vibration prediction of spindles.
\end{abstract}

\section{Introduction}

Ball screw feed drives are frequently used to position the spindle (or stage) to the desired location due to their high stiffness and accuracy. The positioning precision and efficiency directly determine the quality and productivity of machine tools [1]. Hence, it is necessary to possess insight into the effect of ball screw feed drives on the dynamic characteristics of spindles or stages.

It has become mainstream to consider the effect of ball screw feed drives in the study of dynamic characteristics of stages. For instance, taking the stiffness of the ball bearings, the screw shaft, and the screw-nut interface into account, the vibration characteristics of the stage were investigated by means of lumped-parameter method [2-5]. Besides, considering the effects of the preload and rigidity of linear guide modules, the vibration characteristics of the stage were researched using the FE method [6-9]. In the analytical model of a linear feeding stage [10], the rigidity of linear guide modules was also taken into account. However, the rolling interfaces between the guide rail and slider were oversimplified. Briefly, the ball screw feed drives have a strong influence on the dynamic behavior of stages. Nevertheless, the modeling accuracy of the linear guide module requires further improvement.

Research on the dynamic behavior of spindles was conducted using a variety of approaches [11-13]. The dynamic models accounted for the spindle shaft and bearing, the holder and cutter, and the frame structure of machine tools. However, the mechanical characteristics of ball screw feed drives were rarely taken into consideration. Preloading of the ball screw feed drive was considered and the dynamic behavior of a vertical column-spindle system was analyzed by Hung et al. [14, 15]. But, it is time-consuming to obtain the desired results with the FE model. Therefore, further research on the vibration characteristics of spindles under the influences of ball screw feed drives should be conducted.

In order to make a contribution to the vibration prediction of spindles, an analytical modeling approach is proposed to establish a mathematical model for a ball-screwfeeding spindle system (BSFSS). The BSFSS is simplified as a mass-spring system with six degrees of freedom. The rolling interfaces in linear guide modules are emphatically considered and accurately described. The motion equations 


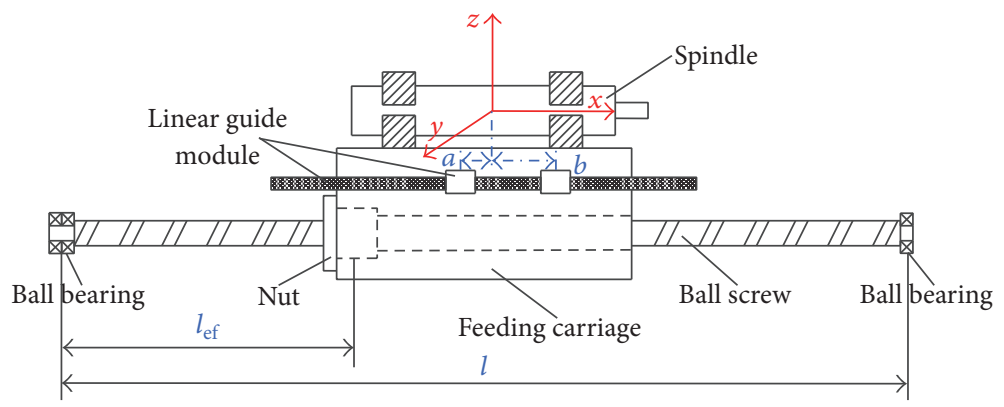

FIgURE 1: Schematic drawing of the BSFSS.

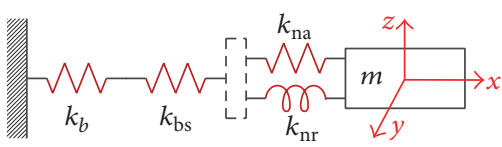

(a)

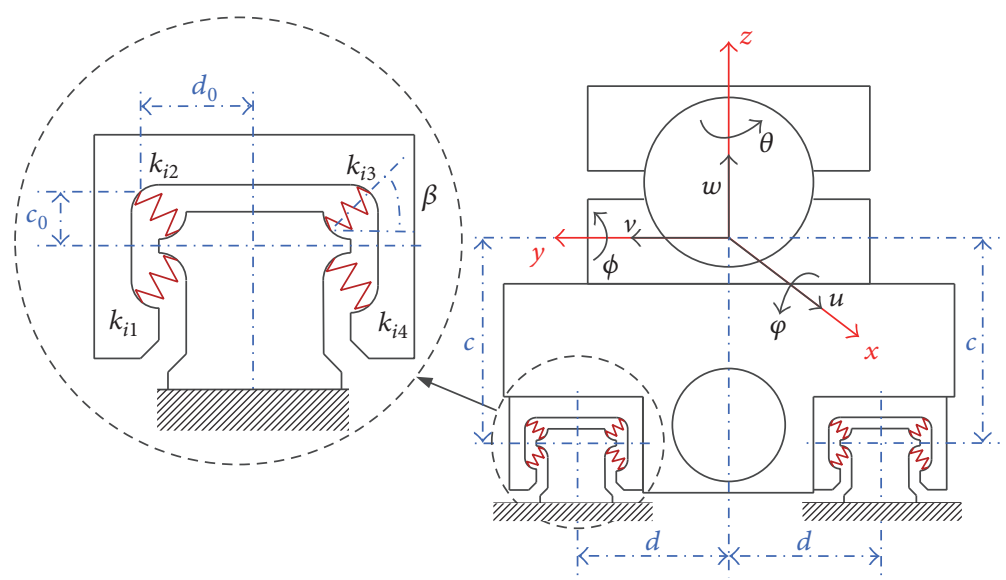

(b)

FIGURE 2: Simplified mechanical model of the BSFSS.

and numerical solutions are derived. The modal experiments are conducted for validation of the presented model. With the proposed mathematical model, parameter-dependent dynamics of the feeding carriage of the spindle (FCoS) is also discussed. Finally, a conclusion is drawn.

\section{Analytical Modeling Approach}

2.1. Dynamic Model. A BSFSS considered here is shown in Figure 1. The spindle is fixed on the feeding carriage. The feeding carriage driven by the ball screw is mounted on the machine base through a pair of linear guides. The ball screw is supported by ball bearings. At the left end, two angular contact ball bearings are mounted back to back to sustain the axial and radial forces. At the right end, one deep groove ball bearing is installed to provide the radial force. The commercial linear guide module (composed of a linear guide and a slider) has four ball grooves with a circular arc profile forming a point contact at the angle of 45 degrees. The linear guide module can be preloaded at a different amount. The preloads are quantified as low preload $(\mathrm{Z0}, 0.01 \mathrm{C})$, medium preload (ZA, $0.06 C)$, and high preload $(\mathrm{ZB}, 0.11 C)$, where $C$ denotes the dynamic load rating $(7.83 \mathrm{kN})[16]$.

The joints between the spindle and feeding carriage also influence the dynamic behavior of the spindle. For simplification, the joint stiffness is assumed to be infinite. And the spindle and feeding carriage are represented together as a rigid body with a total mass of $m$, as depicted in Figure 2(a). The ball bearings at the left end are modeled by a linear elastic connection with axial stiffness $k_{b}$. The radial stiffness of ball bearings mainly determines the bending vibration of the ball screw. In the study of the axial and torsional vibration of the ball screw, the radial stiffness of ball bearings is normally neglected $[5,17,18]$. Inspired by those studies, the radial stiffness of ball bearings is not considered in the model here. The screw shaft is simplified as a linear elastic connection with axial stiffness $k_{\mathrm{bs}}$. The screw-nut interface is endowed with an axial stiffness $k_{\mathrm{na}}$ and a torsional stiffness $k_{\mathrm{nr}}$.

The rigidity of the linear guide module is governed by the contact stiffness of rolling interfaces between rolling balls and grooves because the guide rail and slider are more rigid in structure. Hence, by neglecting the mass of rolling balls, each slider is assumed to be supported by four spring elements in the direction of the contact angle. The spring element is located at the middle of each ball groove longitudinally. As shown in Figure 2(b), the spring elements are quantified with stiffness values $k_{i 1}, k_{i 2}, k_{i 3}$, and $k_{i 4}$ for each linear guide module $(i=1,2,3,4)$.

A coordinate system for vibration motions of the FCoS is illustrated in Figure 2(b). The origin of the coordinate system is the gravity center of the simplified rigid body $m$. The feeding direction of the spindle is along $x$-axis. $c \pm c_{0}$ and 


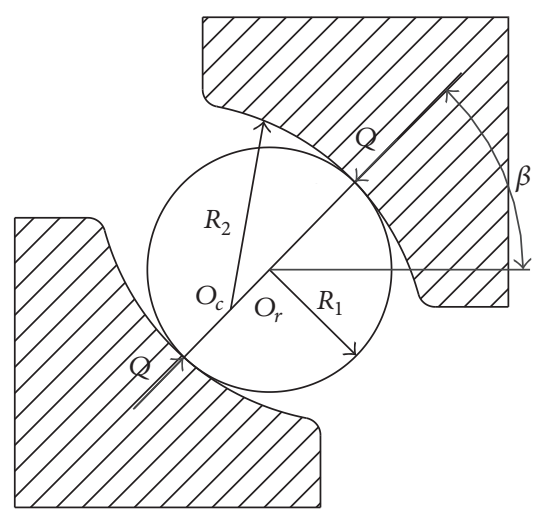

FIGURE 3: Contact model of the ball and grooves.

$d \pm d_{0}$ are the distances from the contact points between the rolling balls and sliders to $x-y$ plane and $x-z$ plane, respectively. As depicted in Figure 1, $a$ and $b$ are defined as the distances from the contact points to $y$ - $z$ plane.

According to the aforementioned simplification, the BSFSS is modeled as a mass-spring system. The vibration modes associated with the motion degrees of freedom can be defined. The translational motions $u, v$, and $w$ are defined as linear displacements in axial, lateral, and vertical directions, respectively. The displacement in the axial direction is called axial vibration mode. The rocking motion $\varphi$ about $x$-axis is known as rolling vibration mode. The rocking motion $\phi$ about $y$-axis is pitching vibration mode and the rocking motion $\theta$ about $z$-axis is yawing vibration mode.

2.2. Contact Stiffness of Rolling Interfaces. In the linear guide module, the stiffness of the spring elements is determined by the contact characteristics of rolling interfaces. The contact stiffness of the rolling interface can be calculated according to the Hertzian theory [19]. As illustrated in Figure 3, $R_{1}$ is the radius of the ball, $R_{2}$ is the radius of grooves in the guide rail and the slider, $\beta$ is the contact angle, and $Q$ denotes the contact force. To enhance the accuracy of the model, the contact is regarded as elastic.

The contact force can be related to the local deformation at the contact point by the Hertzian expression [20]:

$$
Q=k_{h} d^{3 / 2},
$$

where $d$ is the elastic deformation at the contact point. $k_{h}\left[\mathrm{~N} / \mathrm{m}^{3 / 2}\right]$ representing the Hertz constant is determined as

$$
k_{h}=\frac{4 \sqrt{2}}{3 B^{3 / 2} E^{\prime} R^{1 / 2}},
$$

where

$$
\begin{aligned}
E^{\prime} & =\frac{1-u_{1}^{2}}{E_{1}}+\frac{1-u_{2}^{2}}{E_{2}}, \\
R & =\rho_{11}+\rho_{12}+\rho_{21}+\rho_{22}=\frac{1}{R_{1}}+\frac{1}{R_{1}}+0-\frac{1}{R_{2}}, \\
f(\rho) & =\frac{\left|\left(\rho_{11}-\rho_{12}\right)+\left(\rho_{21}-\rho_{22}\right)\right|}{R} .
\end{aligned}
$$

In the above equations, $E^{\prime}\left[\mathrm{m}^{2} / \mathrm{N}\right]$ describes the comprehensive material property of the ball and groove. $E_{1}$ and $E_{2}$ are Young's modulus for materials of the ball and groove, respectively. $u_{1}$ and $u_{2}$ are Poisson's ratios. $R\left[\mathrm{~m}^{-1}\right]$ is the synthetic curvature of the ball and groove. $B$, named as the Hertz coefficient, can be obtained based on the value of $f(\rho)$ [21]. $f(\rho)$ and $B$ are both dimensionless. The contact stiffness $K_{n}$ between the ball and groove can then be obtained using

$$
K_{n}=\frac{\mathrm{dQ}}{\mathrm{d} d}=\frac{3}{2} k_{h} d^{1 / 2}=\frac{3}{2} k_{h}^{2 / 3} \mathrm{Q}^{1 / 3}
$$

As revealed in (4), the contact stiffness depends nonlinearly on the contact force, which is essentially determined by the preload set on the rolling ball.

2.3. Mathematical Model and Analytical Solution. The kinetic energy $T$ regarding the mass and inertia of the system is

$$
\begin{aligned}
T= & \frac{1}{2} m \dot{u}^{2}+\frac{1}{2} m \dot{v}^{2}+\frac{1}{2} m \dot{w}^{2}+\frac{1}{2} J_{x} \dot{\varphi}^{2}+\frac{1}{2} J_{y} \dot{\phi}^{2} \\
& +\frac{1}{2} J_{z} \dot{\theta}^{2} .
\end{aligned}
$$

The potential energy $U$, due to the ball bearing, the ball screw, and the linear guide module, can be written as

$$
\begin{aligned}
U & =\sum_{i=1}^{4} \sum_{j=1}^{4} \frac{1}{2} k_{i j} \delta_{i j}^{2}+\frac{1}{2} k_{\mathrm{eq}} u^{2}+\frac{1}{2} k_{\mathrm{nr}} \varphi^{2} \\
& =\sum_{i=1}^{4} \frac{1}{2}\left[k_{i 1}\left(A_{i 1} \cos \beta+B_{i 1} \sin \beta\right)^{2}\right. \\
& +k_{i 2}\left(A_{i 2} \cos \beta-B_{i 2} \sin \beta\right)^{2} \\
& +k_{i 3}\left(A_{i 3} \cos \beta+B_{i 3} \sin \beta\right)^{2} \\
& \left.+k_{i 4}\left(A_{i 4} \cos \beta-B_{i 4} \sin \beta\right)^{2}\right]+\frac{1}{2} k_{\mathrm{eq}} u^{2}+\frac{1}{2} k_{\mathrm{nr}} \varphi^{2},
\end{aligned}
$$

where

$$
\begin{aligned}
& A_{11}=v+\left(c+c_{0}\right) \varphi+a \theta, \\
& B_{11}=w-\left(d+d_{0}\right) \varphi-a \phi, \\
& A_{12}=v+\left(c-c_{0}\right) \varphi+a \theta, \\
& B_{12}=w-\left(d+d_{0}\right) \varphi-a \phi, \\
& A_{13}=v+\left(c-c_{0}\right) \varphi+a \theta, \\
& B_{13}=w-\left(d-d_{0}\right) \varphi-a \phi, \\
& A_{14}=v+\left(c+c_{0}\right) \varphi+a \theta, \\
& B_{14}=w-\left(d-d_{0}\right) \varphi-a \phi, \\
& A_{21}=v-\left(c+c_{0}\right) \varphi+a \theta, \\
& B_{21}=w+\left(d-d_{0}\right) \varphi-a \phi,
\end{aligned}
$$




$$
\begin{aligned}
& A_{22}=v-\left(c-c_{0}\right) \varphi+a \theta, \\
& B_{22}=w+\left(d-d_{0}\right) \varphi-a \phi, \\
& A_{23}=v-\left(c-c_{0}\right) \varphi+a \theta \text {, } \\
& B_{23}=w+\left(d+d_{0}\right) \varphi-a \phi, \\
& A_{24}=v-\left(c+c_{0}\right) \varphi+a \theta, \\
& B_{24}=w+\left(d+d_{0}\right) \varphi-a \phi, \\
& A_{31}=v-\left(c+c_{0}\right) \varphi-b \theta, \\
& B_{31}=w+\left(d-d_{0}\right) \varphi+b \phi, \\
& A_{32}=v-\left(c-c_{0}\right) \varphi-b \theta, \\
& B_{32}=w+\left(d-d_{0}\right) \varphi+b \phi, \\
& A_{33}=v-\left(c-c_{0}\right) \varphi-b \theta, \\
& B_{33}=w+\left(d+d_{0}\right) \varphi+b \phi \text {, } \\
& A_{34}=v-\left(c+c_{0}\right) \varphi-b \theta, \\
& B_{34}=w+\left(d+d_{0}\right) \varphi+b \phi, \\
& A_{41}=v+\left(c+c_{0}\right) \varphi-b \theta, \\
& B_{41}=w-\left(d+d_{0}\right) \varphi+b \phi \text {, } \\
& A_{42}=v+\left(c-c_{0}\right) \varphi-b \theta, \\
& B_{42}=w-\left(d+d_{0}\right) \varphi+b \phi, \\
& A_{43}=v+\left(c-c_{0}\right) \varphi-b \theta, \\
& B_{43}=w-\left(d-d_{0}\right) \varphi+b \phi, \\
& A_{44}=v+\left(c+c_{0}\right) \varphi-b \theta \text {, } \\
& B_{44}=w-\left(d-d_{0}\right) \varphi+b \phi \text {. }
\end{aligned}
$$

In the above equations, $J_{x}, J_{y}$, and $J_{z}$ are moments of inertia of the simplified rigid body $m$ about $x$-axis, $y$-axis, and $z$-axis, respectively. $\delta_{i j}$ is the displacement of the slider. The subscripts $i=1,2,3,4$ represent the four sliders. The subscripts $j=1,2,3,4$ represent the four rows of grooves in each slider.

Assume that all the linear guide modules have the same specifications and preloaded amounts and that all the vibration motions meet the small displacement assumption. The stiffness of the spring elements can then be presented as

$$
k_{i 1}=k_{i 2}=k_{i 3}=k_{i 4}=k=\frac{K_{n}}{2}, \quad i=1,2,3,4 .
$$

As shown in Figure 2(a), the linear elastic connections $k_{b}$, $k_{\mathrm{bs}}$, and $k_{\mathrm{na}}$ are in series. Hence, the equivalent axial stiffness can be derived as

$$
\frac{1}{k_{\mathrm{eq}}}=\frac{1}{k_{b}}+\frac{1}{k_{\mathrm{bs}}}+\frac{1}{k_{\mathrm{na}}}
$$

where

$$
k_{\mathrm{bs}}=\frac{E \pi D^{2}}{4 l_{\mathrm{ef}}},
$$

with Young's modulus $E$, the diameter $D$, and the equivalent length $l_{\text {ef }}$ of the screw shaft. The torsional stiffness of the screw-nut interface can be obtained using

$$
k_{\mathrm{nr}}=\left(\frac{p}{2 \pi}\right)^{2} k_{\mathrm{na}}
$$

with lead $p$ of the screw shaft.

Applying the Lagrange approach to (5)-(11), the motion equations of the mass-spring system can be derived:

$$
\begin{aligned}
& m \ddot{u}+k_{\mathrm{eq}} u=0, \\
& m \ddot{v}+16 k \cos ^{2} \beta \cdot v+8 k(a-b) \cos ^{2} \beta \cdot \theta=0, \\
& m \ddot{w}+16 k \sin ^{2} \beta \cdot w+8 k(b-a) \sin ^{2} \beta \cdot \phi=0, \\
& J_{x} \ddot{\varphi}+16 k\left[\left(c^{2}+c_{0}^{2}\right) \cos ^{2} \beta+\left(d^{2}+d_{0}^{2}\right) \sin ^{2} \beta\right] \varphi \\
& \quad+k_{\mathrm{nr}} \varphi=0, \\
& J_{y} \ddot{\phi}+8 k\left(a^{2}+b^{2}\right) \sin ^{2} \beta \cdot \phi \\
& \quad+8 k(b-a) \sin ^{2} \beta \cdot w=0, \\
& J_{z} \ddot{\theta}+8 k\left(a^{2}+b^{2}\right) \cos ^{2} \beta \cdot \theta+8 k(b-a) \cos ^{2} \beta \cdot v=0 .
\end{aligned}
$$

It is noticed that the first motion equation governs the displacement $u$ along $x$-axis, and the fourth motion equation describes the angular displacement $\varphi$ about $x$-axis. The natural frequencies including the axial $\left(f_{A}\right)$ and rolling $\left(f_{R}\right)$ modes can be calculated using

$$
\begin{aligned}
f_{A} & =\frac{1}{2 \pi} \sqrt{\frac{k_{\mathrm{eq}}}{m}}, \\
f_{R} & =\frac{1}{2 \pi} \\
& \cdot \sqrt{\frac{16 k\left[\left(c^{2}+c_{0}^{2}\right) \cos ^{2} \beta+\left(d^{2}+d_{0}^{2}\right) \sin ^{2} \beta\right]+k_{\mathrm{nr}}}{J_{x}}} .
\end{aligned}
$$

As revealed in (12), the linear displacement $v$ and the angular displacement $\theta$ are highly coupled. The solution is assumed to be the form

$$
\begin{aligned}
& v=v^{\prime} \cdot e^{j \omega t}, \\
& \theta=\theta^{\prime} \cdot e^{j \omega t} .
\end{aligned}
$$

Substituting (14) into the second and the sixth motion equations of (12), the characteristic equation can be derived as

$$
m J_{z} \omega^{4}-\left(B_{1} J_{z}+B_{2} m\right) \omega^{2}+B_{1} B_{2}-B_{3}^{2}=0,
$$


where

$$
\begin{aligned}
& B_{1}=16 k \cos ^{2} \beta, \\
& B_{2}=8 k\left(a^{2}+b^{2}\right) \cos ^{2} \beta, \\
& B_{3}=8 k(a-b) \cos ^{2} \beta .
\end{aligned}
$$

Similarly, the characteristic equation for the linear displacement $w$ and the angular displacement $\phi$ can also be derived as

$$
m J_{y} \omega^{4}-\left(C_{1} J_{y}+C_{2} m\right) \omega^{2}+C_{1} C_{2}-C_{3}^{2}=0
$$

where

$$
\begin{aligned}
& C_{1}=16 k \sin ^{2} \beta, \\
& C_{2}=8 k\left(a^{2}+b^{2}\right) \sin ^{2} \beta, \\
& C_{3}=8 k(b-a) \sin ^{2} \beta .
\end{aligned}
$$

Finally, the natural frequencies of the yawing $\left(f_{Y}\right)$ and pitching $\left(f_{P}\right)$ modes are computed as

$$
\begin{aligned}
& f_{Y}=\frac{\omega_{1}}{2 \pi}, \\
& f_{P}=\frac{\omega_{2}}{2 \pi},
\end{aligned}
$$

where

$$
\begin{aligned}
& \omega_{1}^{2} \\
& =\frac{B_{1} J_{z}+B_{2} m-\sqrt{\left(B_{1} J_{z}+B_{2} m\right)^{2}-4 m J_{z}\left(B_{1} B_{2}-B_{3}^{2}\right)}}{2 m J_{z}}, \\
& \omega_{2}^{2} \\
& =\frac{C_{1} J_{y}+C_{2} m-\sqrt{\left(C_{1} J_{y}+C_{2} m\right)^{2}-4 m J_{y}\left(C_{1} C_{2}-C_{3}^{2}\right)}}{2 m J_{y}} .
\end{aligned}
$$

The parameters of the BSFSS are either obtained from manufacturers' catalogs or computed from the CAD model of the components, as listed in Table 1. With the low preload set on the linear guide modules $(k=1.9888 \mathrm{~N} / \mu \mathrm{m})$ and the spindle positioned at the middle of its travel range $\left(l_{\mathrm{ef}}=242.5 \mathrm{~mm}\right)$, the natural frequencies associated with the fundamental vibration modes of the FCoS are calculated and listed in Table 2. Substituting the eigenvalues into the characteristic equation, the corresponding eigenvectors can also be derived, which describe the vibration modes. The yawing mode $\left(f_{Y}\right)$ stands for the coupling of the translational motion $v$ and the rocking motion $\theta$, in which the rocking motion $\theta$ is primary. The pitching mode $\left(f_{P}\right)$ expresses the coupling of the translational motion $w$ and the rocking motion $\phi$, where the rocking motion $\phi$ is dominant. The rolling mode $\left(f_{R}\right)$ means the rocking motion $\varphi$, and the axial mode $\left(f_{A}\right)$ represents the translational motion $u$.
TABLE 1: Parameters of the BSFSS.

\begin{tabular}{lcc}
\hline Parameter & Value & Unit \\
\hline$R_{1}$ and $R_{2}$ & $1.389 e-3$ and $1.45 e-3$ & $\mathrm{~m}$ \\
$E_{1}=E_{2}$ & $2.06 e 11$ & $\mathrm{~Pa}$ \\
$u_{1}=u_{2}$ & 0.3 & - \\
$\beta$ & $\pi / 4$ & $\mathrm{rad}$ \\
$E$ & $2.11 e 11$ & $\mathrm{~Pa}$ \\
$l, D$, and $p$ & $0.78,0.016$, and 0.005 & $\mathrm{~m}$ \\
$m$ & 13.7 & $\mathrm{Kg}$ \\
$J_{x}, J_{y}$, and $J_{z}$ & $0.06,0.016$, and 0.12 & $\mathrm{Kg} \cdot \mathrm{m}^{2}$ \\
$k_{b}$ and $k_{\text {na }}$ & $2.45 e 7$ and $1.078 e 8$ & $\mathrm{~N} / \mathrm{m}$ \\
$k$ (low, middle, and high & $1.9888 e 6,3.6139 e 6$, and $4.4231 e 6$ & $\mathrm{~N} / \mathrm{m}$ \\
preload) & 0.0195 and 0.0445 & $\mathrm{~m}$ \\
$a$ and $b$ & 0.093 and 0.0025 & $\mathrm{~m}$ \\
$c$ and $c_{0}$ & 0.0325 and 0.0075 & $\mathrm{~m}$ \\
$d$ and $d_{0}$ & &
\end{tabular}

TABle 2: Predicted and experimental natural frequencies of the BSFSS.

\begin{tabular}{lccc}
\hline $\begin{array}{l}\text { Vibration } \\
\text { mode }\end{array}$ & Prediction/Hz & Experiment/Hz & Relative error/\% \\
\hline Yawing mode & 36.7 & 35.1 & $4.56 \%$ \\
Pitching mode & 86.7 & 81.8 & $5.99 \%$ \\
Rolling mode & 162.1 & 149.1 & $8.72 \%$ \\
Axial mode & 182.0 & 166.3 & $9.44 \%$ \\
\hline
\end{tabular}

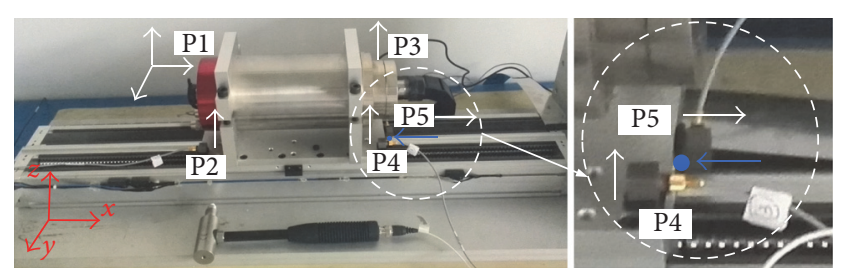

FIgURE 4: Experimental setup.

\section{Experimental Validation and Discussion}

3.1. Modal Test Validation. As depicted in Figure 4, the modal test is conducted on a ball-screw-feeding spindle setup. The linear guide modules are preloaded at low amounts (Z0) and the spindle is positioned at the middle of its travel range. Using the impact testing method, the hammering point is placed on the side (small dot) of the feeding carriage in the opposite direction of $x$-axis. Four accelerometers are arranged at the corners on the upper surface of the feeding carriage. Another accelerometer is located near the hammering point. The accelerometer at point P1 can obtain three-direction acceleration signals. The three accelerometers at points $\mathrm{P} 2, \mathrm{P} 3$, and $\mathrm{P} 4$ can obtain the acceleration signals in the direction of $z$-axis. The last accelerometer at point $\mathrm{P} 5$ can obtain the acceleration signals in the direction of $x$-axis. The directions of the signals are consistent with those of the BSFSS coordinates, and three tests are performed.

With the impulse excitations at point P5, the FRFs of point P1 (in three directions), point P2 (in the direction 


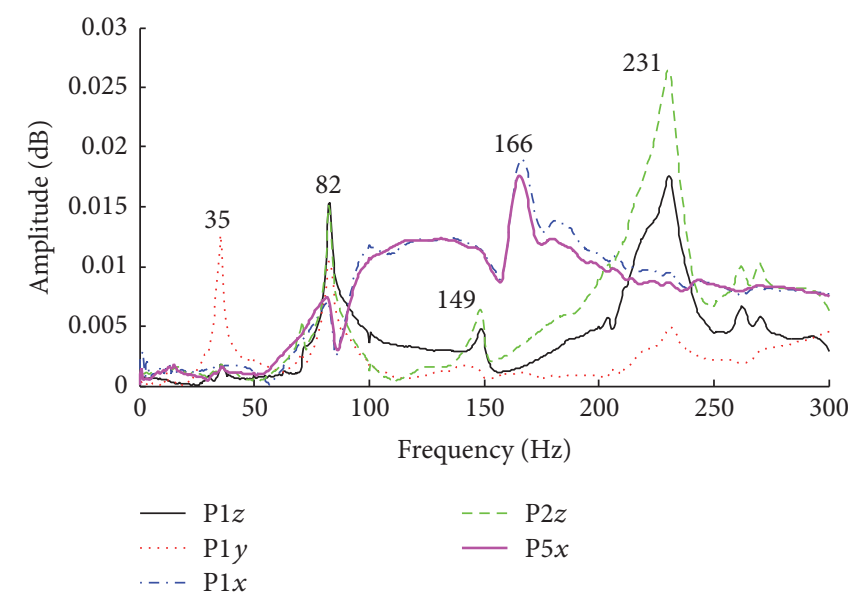

FIGURE 5: FRFs of points P1, P2, and P5.

of $z$-axis), and point P5 (in the direction of $x$-axis) are obtained using the LMS impact testing system. As illustrated in Figure 5, the natural frequency at $35 \mathrm{~Hz}$ stands for the yawing dominant vibration, which excites the acceleration in the direction of $y$-axis. The pitching dominant vibration at $82 \mathrm{~Hz}$ and the rolling dominant vibration at $149 \mathrm{~Hz}$ both induce the acceleration in the direction of $z$-axis. The axial dominant vibration at $166 \mathrm{~Hz}$ arouses the acceleration in the direction of $x$-axis. The coupling vibration of the rigid pitching mode and the flexible bending mode at $231 \mathrm{~Hz}$, which is not contained in the mathematical model, also causes the acceleration in the direction of $z$-axis.

With the FRFs, the dynamic parameters at low frequencies can be estimated. The experimental natural frequencies of different vibration modes are listed in Table 2 for comparison. The maximum relative error between the predicted natural frequency and the experimental natural frequency is $9.44 \%$. According to the LMS modal analysis software, the modal damping ratio of the vibration modes, ranging from $0.83 \%$ to $9.05 \%$, can be estimated. The low damping ratio has little effect on the natural frequency estimation. It can be concluded that the predicted results have a good agreement with the experiment. The deviation may be due to the inaccuracy or ignorance of various stiffness, damping, and inertia.

3.2. Effects of Structural Parameters. According to the proposed mathematical model of the BSFSS, some numerical simulations have been conducted to investigate the parameter-dependent dynamics of the FCoS. Figure 6 depicts the dependence of natural frequencies on the preload set on the linear guide module. This reveals apparent dependence of natural frequencies associated with the yawing and pitching modes on the preload. It is also shown that the preload has a strong influence on the natural frequency corresponding to the rolling mode. For the natural frequency of the axial mode, the preload has no effect at all. The reason is that the axial mode is mainly determined by the stiffness of the ball bearings, the ball screw, and the screw-nut interface rather than the rigidity of the linear guide module.

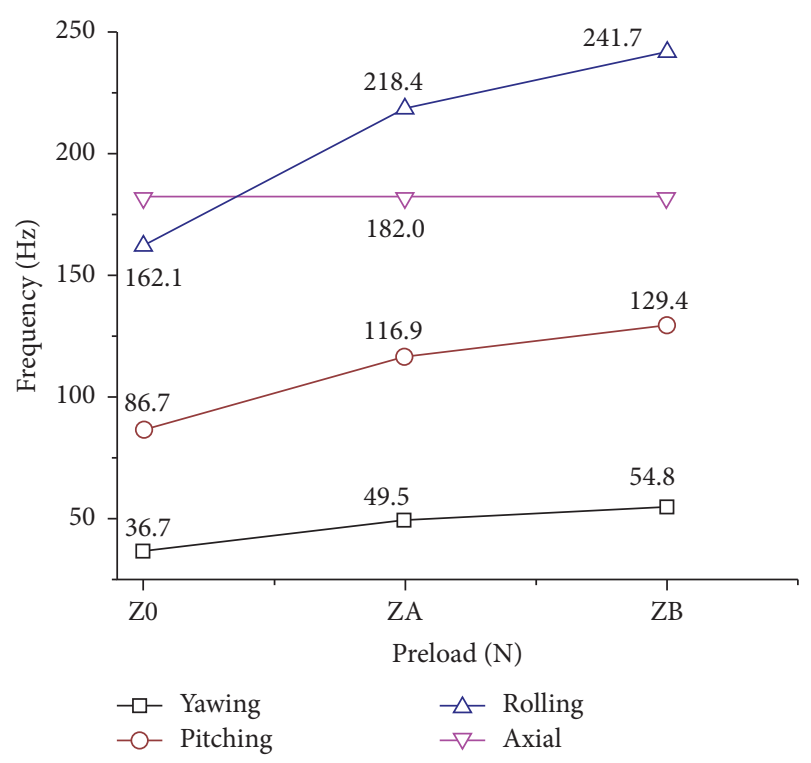

FIGURE 6: Dependence of the natural frequencies on the preload.

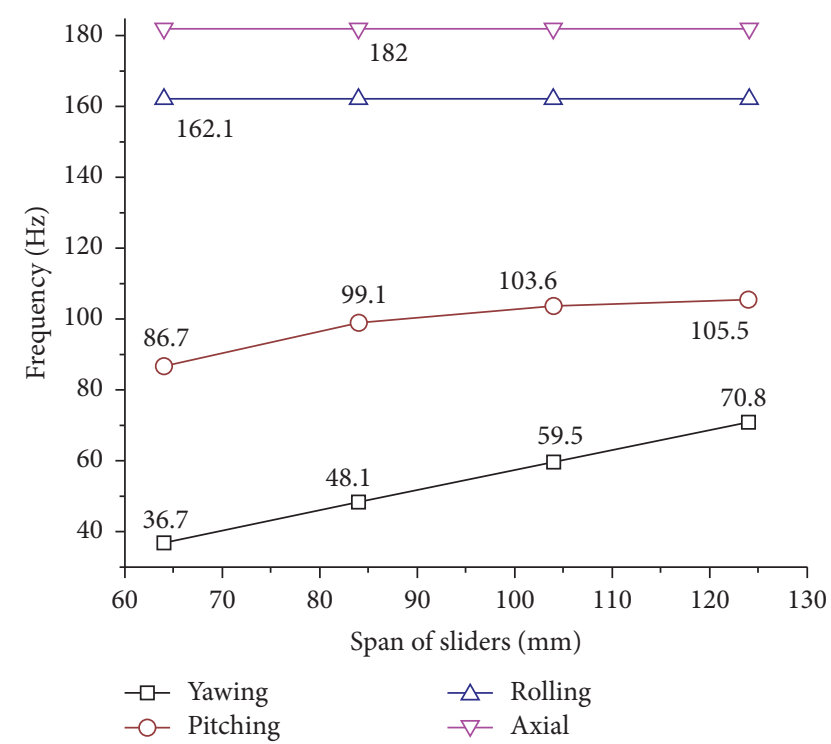

FIGURE 7: Influence of the span of sliders on the natural frequencies.

On the other hand, Figure 7 presents the influence of the span of sliders on the natural frequencies. With the span of sliders increasing from $64 \mathrm{~mm}$ to $124 \mathrm{~mm}$, the yawing natural frequency changes from $36.7 \mathrm{~Hz}$ to $70.8 \mathrm{~Hz}$, and the pitching natural frequency increases to $105.5 \mathrm{~Hz}$ from the original 86.7 Hz. Obviously, the span of sliders has no effect on the natural frequencies of the rolling mode and axial mode.

As observed from the analysis, the dependence of natural frequencies for the $\mathrm{FCoS}$ on various structural parameters is distinct. When the preload and span of sliders change, the maximum changing rates of natural frequencies reach $49.1 \%$ and $21.7 \%$, respectively. Meanwhile, the preload and span of sliders both have no effect on certain natural 
frequencies. Hence, the analysis and obtaining parameterdependent dynamics of the FCoS are useful and meaningful. The selection of ball screw feed drives can be rapidly finalized so that the dynamic characteristics of the FCoS can be optimized in prototyping design.

In the engineering practice, the lateral and vertical deformation of the ball screw, which is neglected in the proposed model, may have some influence on the yawing and pitching vibrations of the FCoS, especially with the FCoS feeding along the ball screw. Hence, the bending deformation of the ball screw will be additionally considered in the future work.

\section{Conclusions}

Mainly considering the rolling interfaces in the linear guide modules, a mathematical model of the BSFSS is derived. The predicted results correspond closely with the experimental data. The ball screw feed drive, especially the linear guide module, is shown to determine the vibration behavior of the FCoS. The bending deformation of the ball screw and the bearing stiffness of the spindle will be additionally considered to predict the dynamic behavior of spindles.

With the mathematical model, the vibration behavior of the FCoS is rapidly analyzed for various structural parameters. This will be helpful for optimizing the structural parameters of ball screw feed drives in prototyping design.

\section{Competing Interests}

The authors declare that there are no competing interests regarding the publication of this paper.

\section{Acknowledgments}

The authors would like to thank Mr. Guofeng Wang for his help in design and manufacturing of the experimental setup. And this work was supported in part by National Natural Science Foundation of China (Grant no. 51475324) and Natural Science Foundation of Tianjin (Grant no. 13JCZDJC34000).

\section{References}

[1] Y. Altintas, A. Verl, C. Brecher, L. Uriarte, and G. Pritschow, "Machine tool feed drives," CIRP Annals-Manufacturing Technology, vol. 60, no. 2, pp. 779-796, 2011.

[2] R. Wang, T. Zhao, P. Ye, and Y. Liu, "Three-dimensional modeling for predicting the vibration modes of twin ball screw driving table," Chinese Journal of Mechanical Engineering (English Edition), vol. 27, no. 1, pp. 211-218, 2014.

[3] S. Frey, A. Dadalau, and A. Verl, "Expedient modeling of ball screw feed drives," Production Engineering, vol. 6, no. 2, pp. 205211, 2012.

[4] H. J. Zhang, J. Zhang, H. Liu, T. Liang, and W. H. Zhao, "Dynamic modeling and analysis of the high-speed ball screw feed system," Proceedings of the Institution of Mechanical Engineers, Part B: Journal of Engineering Manufacture, vol. 229, no. 5, pp. 870-877, 2015.
[5] Y. Zhou, F. Y. Peng, and X. H. Cao, "Parameter sensitivity analysis of axial vibration for lead-screw feed drives with timevarying framework," Mechanika, vol. 17, no. 5, pp. 523-528, 2011.

[6] L. Mi, G.-F. Yin, M.-N. Sun, and X.-H. Wang, "Effects of preloads on joints on dynamic stiffness of a whole machine tool structure," Journal of Mechanical Science and Technology, vol. 26, no. 2, pp. 495-508, 2012.

[7] D. Wang, Y. Lu, T. Zhang, K. Wang, and A. Rinoshika, "Effect of stiffness of rolling joints on the dynamic characteristic of ball screw feed systems in a milling machine," Shock and Vibration, vol. 2015, Article ID 697540, 2015.

[8] Y.-L. Lai and J.-P. Hung, "Influence of linear guide arrangement on the vibration characteristics of a positioning stage," Journal of the Chinese Society of Mechanical Engineers, vol. 33, no. 2, pp. 113-122, 2012.

[9] W. Sun, X. Kong, and B. Wang, "Precise finite element modeling and analysis of dynamics of linear rolling guideway on supporting direction," Journal of Vibroengineering, vol. 15, no. 3, pp. 1330-1340, 2013.

[10] J.-C. Chang and J.-P. Hung, "Analytical and finite element modeling of the dynamic characteristics of a linear feeding stage with different arrangements of rolling guides," Mathematical Problems in Engineering, vol. 2014, Article ID 454156, 11 pages, 2014.

[11] A. Ertürk, H. N. Özgüven, and E. Budak, "Analytical modeling of spindle-tool dynamics on machine tools using Timoshenko beam model and receptance coupling for the prediction of tool point FRF," International Journal of Machine Tools and Manufacture, vol. 46, no. 15, pp. 1901-1912, 2006.

[12] J.-P. Hung, Y.-L. Lai, T.-L. Luo, and H.-C. Su, "Analysis of the machining stability of a milling machine considering the effect of machine frame structure and spindle bearings: experimental and finite element approaches," International Journal of Advanced Manufacturing Technology, vol. 68, no. 9-12, pp. 2393-2405, 2013.

[13] C.-W. Lin, Y.-K. Lin, and C.-H. Chu, "Dynamic models and design of spindle-bearing systems of machine tools: a review," International Journal of Precision Engineering and Manufacturing, vol. 14, no. 3, pp. 513-521, 2013.

[14] C. Yuan Lin, J. Pin Hung, and T. Liang Lo, "Effect of preload of linear guides on dynamic characteristics of a vertical columnspindle system," International Journal of Machine Tools and Manufacture, vol. 50, no. 8, pp. 741-746, 2010.

[15] J.-P. Hung, Y.-L. Lai, C.-Y. Lin, and T.-L. Lo, "Modeling the machining stability of a vertical milling machine under the influence of the preloaded linear guide," International Journal of Machine Tools and Manufacture, vol. 51, no. 9, pp. 731-739, 2011.

[16] Hiwin Technologies Company, Hiwin Linear Guideway Technical Information, Hiwin Company, Hiwin, Taiwan, 2000.

[17] D. A. Vicente, R. L. Hecker, F. J. Villegas, and G. M. Flores, "Modeling and vibration mode analysis of a ball screw drive," International Journal of Advanced Manufacturing Technology, vol. 58, no. 1-4, pp. 257-265, 2012.

[18] B. Henke, O. Sawodny, and R. Neumann, "Distributed parameter modeling of flexible ball screw drives using ritz series discretization," IEEE/ASME Transactions on Mechatronics, vol. 20, no. 3, pp. 1226-1235, 2015.

[19] D. E. Brewe and B. J. Hamrock, "Simplified solution for elliptical-contact deformation between two elastic solids," Journal of Lubrication Technology, vol. 99, no. 4, pp. 485-487, 1977. 
[20] J. A. Greenwood, "Analysis of elliptical Hertzian contacts," Tribology International, vol. 30, no. 3, pp. 235-237, 1997.

[21] S. Dai, Manual Machine Rolling Bearings Application Handbook, Mechanical Industry Press, Beijing, China, 1993. 


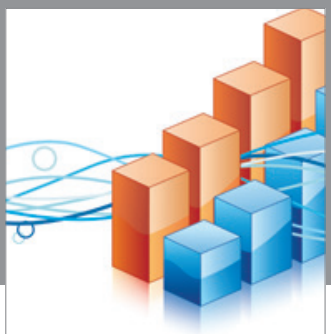

Advances in

Operations Research

vatem alat4

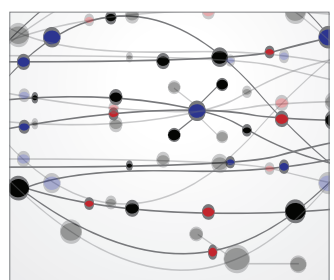

\section{The Scientific} World Journal
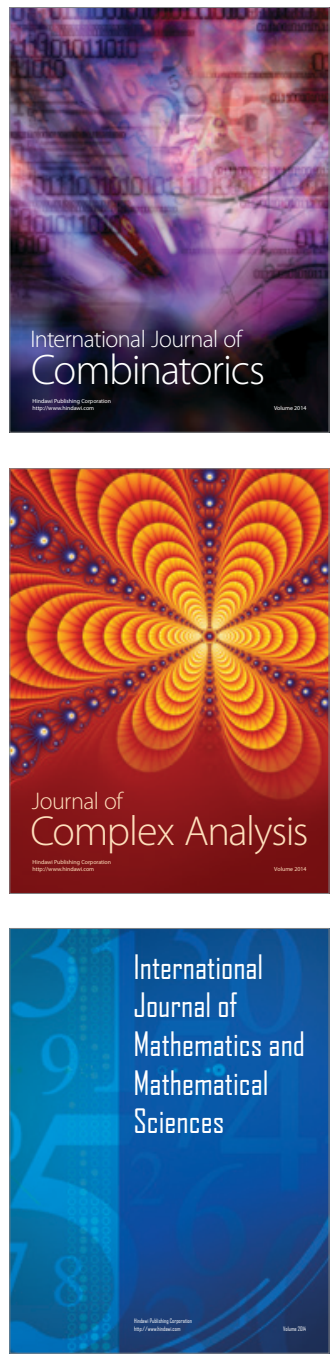
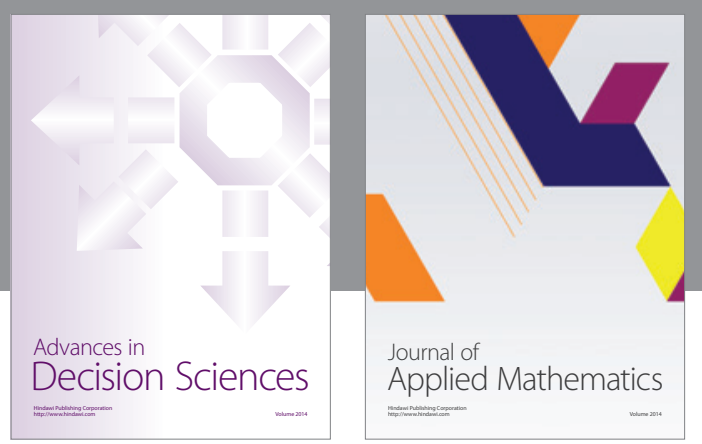

Algebra

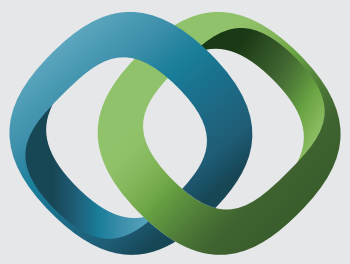

\section{Hindawi}

Submit your manuscripts at

http://www.hindawi.com
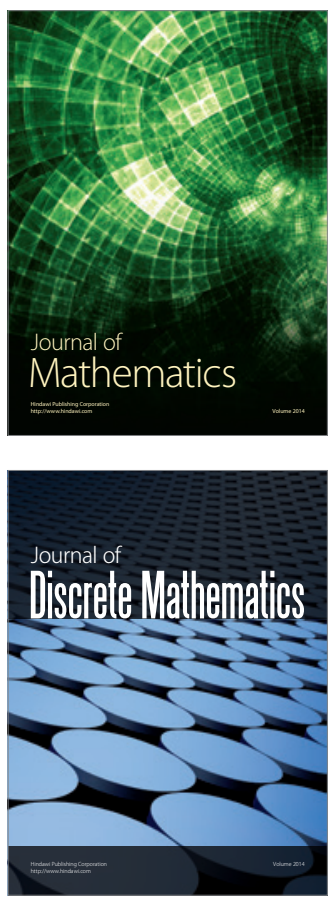

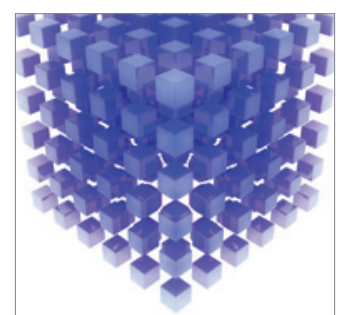

Mathematical Problems in Engineering
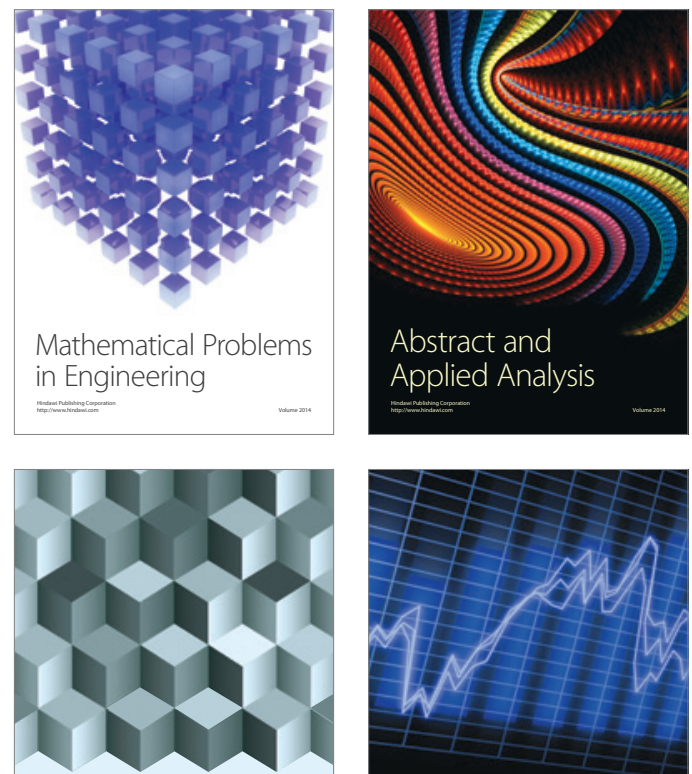

Journal of

Function Spaces

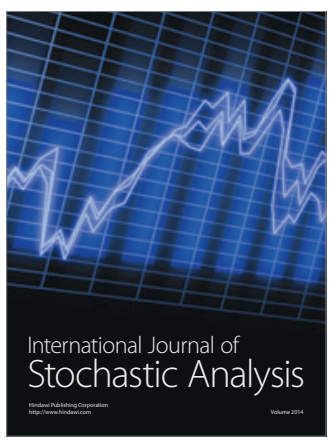

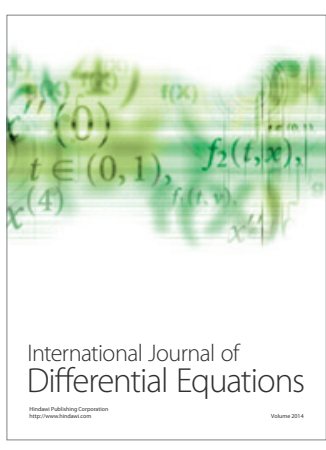
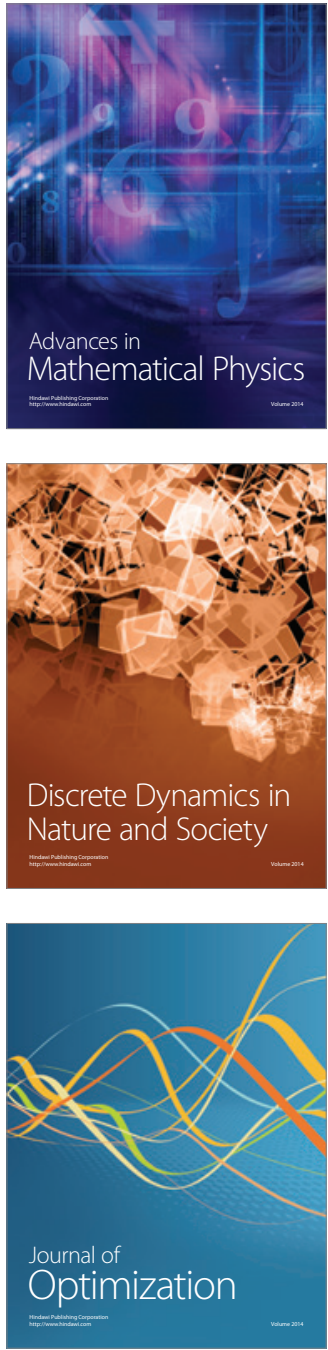